\title{
INVESTIGACIONES
}

\section{Percepción de la calidad de vida en personas con discapacidad y su relación con la educación*}

\author{
Perception of the quality of life of people with disabilities \\ and its relationship with education
Percepção da qualidade de vida das pessoas com deficiência e sua relação com a educação

\author{
Lilian Castro, ${ }^{a}$ José Antonio Casas, Sergio Sánchez, ${ }^{b}$ Viviana Vallejos, Daniela Zúñiga \\ ${ }^{a}$ Universidad de Concepción, Chile \\ Correo electrónico: lilicastro@udec.cl \\ ${ }^{\text {b} U n i v e r s i d a d ~ C a t o ́ l i c a ~ d e ~ M u r c i a, ~ E s p a n ̃ a ~}$ \\ Correo electrónico: ssanchez@ucam.edu
}

\begin{abstract}
RESUMEN
Este trabajo tiene como objetivo principal identificar la percepción que poseen profesorado y alumnado sobre la calidad de vida de los estudiantes con discapacidad de centros de formación laboral, y más concretamente, si existe relación entre la percepción del profesorado y la percepción del alumnado. Para ello, han participado 751 estudiantes y 82 profesionales. Se ha utilizado la Escala Integral de Calidad de Vida. Los principales resultados demuestran que las diferentes dimensiones de calidad de vida percibida por los profesionales se relacionan con sus homólogas en la versión subjetiva, pero además se complementan con dimensiones que atiendan a diversos aspectos de la persona. Los autores concluyen que los resultados encontrados muestran la necesidad de formar a educadores en aspectos relacionados con una educación integral y con metodologías docentes que sirvan para lograr una educación inclusiva.
\end{abstract}

Palabras clave: calidad de vida, discapacidad, profesores, formación.

\section{ABSTRACT}

This paper identifies the perceptions of teachers and students about the quality of life of students with disabilities in vocational training centers. It specifically identifies the correlations between the perceptions of teachers and the perceptions of pupils. A total of 751 students and 82 professionals have participated. The Comprehensive Quality of Life Scale was used. The main results show that the different dimensions of quality of life perceived by professionals interact with their counterparts in the subjective version, but also complement dimensions that address various aspects of the person. The authors conclude that the results show the need to train educators in issues related to comprehensive education and teaching methodologies to achieve inclusive education.

Key words: quality of life, disability, teachers, training.

Proyecto FONDECYT de Iniciación N 11121216 "Calidad de vida en jóvenes con discapacidad intelectual y su relación con la formación laboral”. Apoyo Convenio de desempeño UCO 1203. 


\section{INTRODUCCIÓN}

En la última década, la calidad de vida en las personas con discapacidad, especialmente en las personas con discapacidad intelectual y del desarrollo, ha generado gran interés. Así, se han llevado a cabo múltiples trabajos con el objetivo de proveer planes de apoyos adecuados en diversos ámbitos del desarrollo de la persona con discapacidad (Alomar y Cabre, 2005; Jordán de Urríes y Verdugo, 2003; Luckasson et al., 1992; Peralta, 2008; Thompson et al., 2003; Verdugo, Gómez y Arias, 2007, 2009).

Debido a este interés, en la actualidad coexisten diversos modelos que intentan explicar la calidad de vida de este colectivo (Verdugo et al., 2010). Sin embargo, el modelo propuesto por Schalock y Verdugo $(2002 / 2003,2007,2008)$ aparece en la literatura científica como el más citado y con un mayor número de evidencias científicas que avalan su validez (e.g., Gómez et al., 2011; Verdugo et al., 2011; Wang et al., 2010). Por lo tanto, siguiendo con lo propuesto por Verdugo et al. (2010), este modelo de calidad de vida se define como un estado deseado de bienestar personal que: (a) es multidimensional; (b) tiene propiedades universales (etic) y ligadas a la cultura (emic); (c) tiene componentes objetivos y subjetivos; y (d) está influenciado por características personales y factores ambientales. (Schalock y Verdugo, 2002/2003, 2007, 2008; Verdugo, 2006).

De esta manera, es importante destacar que el modelo multidimensional de Schaclok y Verdugo (2003) describe una calidad de vida basada en ocho dimensiones. Estas dimensiones son: relaciones interpersonales, autodeterminación, inclusión social, bienestar material, bienestar emocional y físico, bienestar laboral, derechos (Schalock y Verdugo, 2002/2003).

A pesar, como se ha descrito anteriormente, de la existencia de numerosos trabajos que estudian la calidad de vida en personas con discapacidad, desafortunadamente, son un porcentaje inferior aquellos trabajos que se han preocupado de realizar un análisis de la contribución que la educación puede traer a dicho colectivo; y cómo esta afecta a la percepción de calidad de vida (Gómez-Vela y Verdugo, 2004). Incluso algunos autores afirman que "se está descuidando enormemente la reflexión compartida, pero, sobre todo, las acciones para que la educación desempeñe el papel crítico que le corresponde en la preparación para una mejor calidad de vida de las personas con discapacidad" (Echeita y Simón, 2007: 3).

Desde la perspectiva de la educación inclusiva (Booth y Ainscow, 2002), se debe realizar especial hincapié en analizar la situación de las personas con discapacidad, para así poder entender su calidad de vida y si este colectivo tiene un acceso igualitario a los procesos de formación (Seda, 2008). Porque, si bien es cierto las personas con discapacidad tienen garantizada su participación en la educación básica (Coll y Miras, 2001; Echeita, 2001; Martín y Mauri, 1997; Martínez, De Haro y Escarbajal, 2010), la situación en otras etapas educativas es muy diferente (Sánchez, 2013). Se estima que en la educación obligatoria la participación de las personas con discapacidad es del $75 \%$ (Echeita y Simón, 2007), sin embargo estos datos son muy diferentes en etapas superiores. Como describe el estudio sobre la situación de las personas con discapacidad en los estados iberoamericanos, estos datos pueden variar en función de diversos motivos (Samaniego, 2009). Entre dichos motivos podemos destacar: “(a) Subsiste la variabilidad en cuanto a conceptos de medición, puntajes y estrategias; y (b) hay diferencias marcadas en cuanto a datos obtenidos, dependiendo si estos fueron tomados por un censo o por una encuesta" (Samaniego, 2009: 178). 
Si el análisis se centra en población chilena, en este mismo informe se describe que existe un 12,9\% de personas con discapacidad en Chile; y de este porcentaje es más alta la población de mujeres respecto de la de hombres. Pero más interesante es el dato que describe que cerca del $90 \%$ de las personas con discapacidad del país tienen más de 30 años (Samaniego, 2009). Es, por lo tanto, una población que ya no se engloba en una educación básica, mientras que si desea recibir una formación complementaria esta se deberá llevar a cabo en ámbitos de la educación universitaria o educación profesionalizante.

Según el informe de la Red Intergubernamental Iberoamericana de Cooperación para la Educación de Personas con Necesidades Educativas Especiales, RIINEE, (2008 Cit. en Samaniego, 2009) la oferta inclusiva que existe en Chile solo se cumple bajo ciertas circunstancias. Entre estas condiciones, se podrían destacar: (a) capacitación durante la universidad a los futuros maestros con mallas curriculares que sirvan para atender a la diversidad; (b) capacitación a los maestros en activo; (c) disminución de la ratio de alumnos con discapacidad - permanentes y temporales - en el aula; (d) ofertar ayudas técnicas para apoyar la docencia; y (e) proporcionar herramientas para adaptar el material necesario en las clases. Sin embargo, a pesar de disponer de toda una batería de medidas que puedan garantizar la inclusión educativa, se observa en base a la anterior descripción que la mayoría de las medidas siguen estando enfocadas en la educación básica o elemental.

Desgraciadamente, esto no es una situación aislada en Chile, ya que es menos frecuente la existencia de actuaciones - y sobre todo de informes técnicos rigurosos- que avalen el uso de medidas estandarizadas para atender a la diversidad en etapas no obligatorias (Sánchez, 2013). La situación de la universidad es algo menos preocupante, ya que diversas entidades disponen de recursos para dar soporte a las personas con discapacidad en esta etapa educativa. De este modo, se pueden destacar la Association on higher Education and Disability, que ha publicado unos estándares y protocolos de atención a las personas con discapacidad (Dukes, 2001, 2006), la European Association for Quality Assurance in Higher Education, que describió unos criterios y directrices para la garantía de calidad en el Espacio Europeo de Educación Superior (2005), o la reciente publicación de un documento sobre estándares e indicadores de buenas prácticas para la atención a estudiantes universitarios con discapacidad en España (Díez et al., 2011).

Aunque si hablamos de formación superior fuera del ámbito universitario, es prácticamente inexistente el estudio de la educación al colectivo de personas con discapacidad y la manera en que se ven atendidos sus derechos en la formación profesional que reciben (Pallisera, Fullana y Vila, 2010). Por tanto, este hecho debe ser reclamado por los centros de investigación para así atender a las obligaciones que se expresan en la ley. Y es que el Artículo 5, en el aparatado de las disposiciones generales de la III.1.Ley $\mathrm{N}^{\circ} 19.284$ que establece las normas para la plena integración social de personas con discapacidad, se dice lo siguiente: "El sistema escolar en su conjunto deberá ofrecer opciones educativas a través de diferentes modelos de integración escolar en todos los niveles del sistema: prebásico, básico, medio humanístico científico o técnico profesional y superior.” (González y Araneda, 2005: 15).

Por otro lado, retomando la calidad de vida desde el punto de vista de las propias personas con discapacidad y especialmente en las personas con DID, diversos instrumentos han sido desarrollados con una doble visión (Gómez et al., 2011). Por un lado, la perspectiva de los cuidadores o personas de referencia en la vida de este colectivo y, por otro lado, el de las personas con discapacidad como principal agente de evaluación de su propia calidad, este es el caso de la Escala Integral (Verdugo et al., 2009). Esta escala evalúa la 
calidad de vida desde dos perspectivas: objetiva y subjetiva. La parte objetiva es aplicada por los profesionales que trabajan con la persona con discapacidad, mientras que la escala subjetiva se centra en la propia percepción de la persona con discapacidad sobre su calidad de vida en diversas dimensiones. Y es que no se puede obviar que la voz de las personas con discapacidad debe ser escuchada (Echeita, 2008). Diversos estudios en el ámbito de educación superior se han centrado en conocer las percepciones de los estudiantes, normalmente estudiantes con discapacidad, acerca de su situación en el proceso de enseñanza y aprendizaje en entornos educativos (Castellana y Sala, 2005). Son los propios participantes los que deben describir cuál es la situación en la que se encuentran en un proceso de búsqueda de la mejora de la denominada educación inclusiva (Echeita, 2008).

A nivel internacional, destacan fundamentalmente las investigaciones centradas en conocer las percepciones de los estudiantes acerca de posibles medidas que favorecen o eliminan posibilidades en la inclusión de los colectivos más desfavorecidos en la educación superior (Burgstahler, 2006; Burgstahler y Cory, 2008; Schelly, Davies y Spooner, 2011). En un estudio llevado a cabo por Castellana y Sala (2005) se examinan las necesidades y dificultades que encuentran los estudiantes de niveles superiores con discapacidad dentro del aula, y las necesidades y dificultades que el profesor tiene para atender a estos estudiantes en el contexto del aula. Y es este último aspecto, las destrezas por parte del profesorado, una característica importante a tener en cuenta en aquellos profesionales que se dedican a la formación de las personas con discapacidad (Belmonte y Suñe, 2014).

Como describen algunos autores (Castellana y Sala, 2005), la percepción de los estudiantes con discapacidad por sentirse incluidos y aceptados en el aula es la principal demanda que reclama este colectivo. Además, la falta de conocimientos sobre la discapacidad es el principal obstáculo que los profesores encuentran para poder dar respuesta a las necesidades de las personas con discapacidad (Gandasegui y Méndez, 2014; Pallisera, 2011).

Por tanto, esta investigación tiene como objetivo principal identificar la percepción, para una posterior mejora de los planes de formación de los profesionales, que tiene tanto el profesorado como el propio alumnado sobre la calidad de vida de sus estudiantes con discapacidad de centros de formación superior, más concretamente de formación laboral, de la VIII Región del Biobío, Chile.

\section{MÉTODO}

Este estudio instrumental se ha llevado a cabo mediante un diseño prospectivo, transversal, ex post facto (Montero y León, 2007).

\subsection{PARTICIPANTES}

Ha participado un total de 751 estudiantes con DID inmersos en un programa de formación laboral, pertenecientes a talleres laborales de Escuelas Especiales de la Región del Biobío. También 82 profesionales a cargo de tutorizar y formar a los estudiantes. De la totalidad de los jóvenes, 293 son de género femenino (39.01\%) y 458 de género masculino (60.99\%). Con una edad comprendida entre los 14 y los 55 años, $(M=22.87$; DT = 5.86). Participan estudiantes de 30 establecimientos de los 32 existentes en la Región del Biobío. 


\subsection{INSTRUMENTO}

Se ha utilizado la Escala Integral (Verdugo et al., 2009) validada en población chilena (Castro et al., 2016). Esta escala evalúa la calidad de vida desde dos perspectivas, objetiva y subjetiva, en adultos con DID. Así, la Escala Objetiva se administra a los profesionales que trabajan con la persona con discapacidad y muestra su valoración de forma externa. La Escala Subjetiva refleja la valoración y percepción de la propia persona con discapacidad.

La Escala Objetiva está compuesta por 23 ítems divididos en 5 dimensiones: 1) Autodeterminación, 8 ítems que miden la percepción del profesional sobre la autodeterminación de la persona con discapacidad, con ítems, como por ejemplo: Manifiesta intereses y objetivos propios y hace planes para alcanzarlos. 2) Inclusión social, 3 ítems que evalúan la inclusión con ítems como Tiene amigos con los que realizar actividades de su agrado. 3) Bienestar Laboral, de 3 ítems como Realiza su trabajo de forma competente y responsable. 4) Bienestar Material, de 4 ítems con ítems como Tiene las cosas que necesita. 5) Bienestar Emocional y Físico, de 5 ítems como Habitualmente está alegre y de buen humor. Estos ítems son de carácter dicotómico y el profesional los evalúa con 0 si no se adquiere y 1 si se han adquirido. Se evalúa cada una de las dimensiones que se componen con la suma de las puntuaciones de cada uno de los ítems que integran las dimensiones evaluadas.

La Escala subjetiva está compuesta por las mismas dimensiones, y además se suma la dimensión de Bienestar Familiar; seis dimensiones en total. Posee un total de 39 ítems tipo Likert de 1 a 4 puntos, ante lo cual se responde de acuerdo a la alternativa que lo representa de mejor forma frente al enunciado planteado: "totalmente en desacuerdo" (1 punto), "en desacuerdo" (2 puntos), "de acuerdo" (3 puntos), "totalmente de acuerdo" (4 puntos). Con una evaluación de suma de puntos al igual que la escala objetiva. La dimensión de autodeterminación, compuesta por 8 ítems como por ejemplo hago compras en tiendas y supermercados. Bienestar laboral, de 4 ítems como mis relaciones en el trabajo son buenas. Bienestar material, de 9 ítems como tengo dinero para comprarme las cosas que necesito. Bienestar emocional y físico, de 7 ítems como Estoy contento con mi vida. Y Bienestar Familiar, de 3 ítems como tengo una buena relación con mis padres.

\subsection{PROCEDIMIENTO}

Se seleccionó a los estudiantes diagnosticados con DID que contaran con habilidades expresivas y comprensivas y a los profesionales a cargo de ellos y ellas, con el requisito que los conocieran al menos 3 meses previo a la fecha de aplicación de la escala. Se solicitaron todos los permisos oportunos a los centros de formación laboral y su aplicación se realizó sobre la base del consentimiento informado de los padres de los jóvenes. La aplicación de las escalas se realizó por parte de miembros del equipo de investigación debidamente formados para ello.

\subsection{ANÁLISIS DE DATOS}

En primer lugar, se han realizado los análisis descriptivos univariados de las dimensiones evaluadas en la escala integral, mediante sus dos componentes: la Escala Objetiva y la Escala Subjetiva. En segundo lugar, se han efectuado 5 modelos de regresión 
Estudios Pedagógicos XLII, Nº 2: 39-49, 2016

PERCEPCIÓN DE LA CALIDAD DE VIDA EN PERSONAS CON DISCAPACIDAD Y SU RELACIÓN CON LA EDUCACIÓN

lineal múltiple mediante el método de pasos sucesivos para comprobar la predicción de las dimensiones evaluadas en la escala subjetiva sobre la percepción del profesorado, evaluando las dimensiones de la calidad de vida de los estudiantes.

\section{RESULTADOS}

Los análisis descriptivos univariados (Tabla 1) muestran los valores alcanzados en todas las variables de la escala integral, tanto en la dimensión objetiva como subjetiva.

Tabla 1. Valores variables escala integral

\begin{tabular}{|l|c|c|c|c|c|}
\hline & N & M & D. T. & Asimetría & Curtosis \\
\hline Objetiva Autodeterminación & 751 & 4,84 & 2,265 & $-1,684$ &,- 881 \\
\hline Objetiva Inclusión Social & 751 & 2,54 &, 804 & $-1,742$ & 2,184 \\
\hline Objetiva Bienestar laboral & 751 & 2,46 &, 841 & $-1,509$ & 1,420 \\
\hline Objetiva Bienestar Material & 751 & 2,42 & 1,369 &,- 278 & $-1,218$ \\
\hline Objetiva Bienestar Emocional y físico & 751 & 4,14 & 1,246 &,- 282 & 1,001 \\
\hline Subjetiva Autodeterminación & 751 & 24,72 & 4,430 & $-1,363$ &, 078 \\
\hline Subjetiva Inclusión social & 751 & 25,86 & 3,960 &,- 570 &, 545 \\
\hline Subjetiva Bienestar Laboral & 751 & 14,76 & 1,809 &,- 666 & 2,748 \\
\hline Subjetiva Bienestar Material & 751 & 31,99 & 3,710 & $-1,022$ &, 718 \\
\hline Subjetiva Bienestar Emocional y físico & 751 & 24,76 & 3,652 & $-1,520$ & 2,346 \\
\hline Subjetiva Bienestar Familiar & 751 & 10,56 & 1,998 & $-1,653$ & 2,539 \\
\hline
\end{tabular}

En segundo lugar, se han realizado modelos de regresión lineal donde las variables dependientes han sido las cinco variables puntuadas por los profesionales al cargo de los estudiantes.

Mediante el procedimiento de regresión por pasos sucesivos encontramos el mejor modelo predictor de la Autodeterminación Objetiva (AutObj) $\left(\mathrm{F}_{4,750}=14.98, \mathrm{p}=.00\right.$ ) obteniéndose un valor de Regresión Múltiple o $\mathrm{R}^{2}$ corregida de .27. Las variables predictivas que entran a formar parte de la ecuación son Autodeterminación Subjetiva (AutSub) $(\mathrm{t}=4.76$; $\mathrm{p}=.00)$; Bienestar emocional y físico subjetivo (BEFSub) $(\mathrm{t}=-3.98 ; \mathrm{p}=.00)$; Inclusión social Subjetiva (ISS) $(\mathrm{t}=3.80 ; \mathrm{p}=.00)$ y Bienestar Familiar Subjetivo (BFS) $(\mathrm{t}=-2.33 ; \mathrm{p}=.02)$

$$
\text { AutObj }=3.68+.18 \text { AutSub }-.15 \text { BEFSub }+.15 \mathrm{ISS}-.09 \mathrm{BFS}
$$

Para el modelo predictor de la Inclusión social Objetiva (ISSOb) $\left(\mathrm{F}_{1,750}=11.07, \mathrm{p}=.01\right)$ se obtuvo un valor de Regresión Múltiple o $\mathrm{R}^{2}$ corregida de .12, en tanto la única variable 
predictiva que entra a formar parte de la ecuación es la Inclusión social Subjetiva (ISS) $(\mathrm{t}=3.32 ; \mathrm{p}=.00)$

$$
\mathrm{ISSOb}=1.90+.12 \mathrm{ISS}
$$

En cuanto a la variable Bienestar laboral objetivo (BLOb) el mejor modelo hallado $\left(\mathrm{F}_{1,750}=31.77, \mathrm{p}=.00\right)$ obtiene un valor de Regresión Múltiple o $\mathrm{R}^{2}$ corregida de .20; la única variable predictiva que entra a formar parte de la ecuación es el Bienestar Laboral Subjetivo (BLSub) $(\mathrm{t}=5.63 ; \mathrm{p}=.00)$

$$
\mathrm{BLOb}=1.07+.20 \mathrm{BLSub}
$$

El mejor modelo predictor del Bienestar Material Objetivo $(\mathrm{BMObj})\left(\mathrm{F}_{3,750}=12.11\right.$, $\mathrm{p}=.00)$ obtiene un valor de Regresión Múltiple o $\mathrm{R}^{2}$ corregida de .21 ; las variables predictivas que entran a formar parte de la ecuación son Autodeterminación Subjetiva (AutSub) ( $\mathrm{t}=3.99 ; \mathrm{p}=.00)$; Bienestar Material Subjetivo (BMSub) $(\mathrm{t}=2.88 ; \mathrm{p}=.00)$ y Bienestar Familiar Subjetivo (BFS) $(\mathrm{t}=-2.01 ; \mathrm{p}=.04)$ :

$$
\mathrm{BMObj}=.55+.15 \mathrm{AutSub}+.11 \mathrm{BMSub}-.07 \mathrm{BFS}
$$

Por último, el modelo realizado para la variable Bienestar emocional y físico objetivo $(\mathrm{BEFObj})\left(\mathrm{F}_{2,750}=25.95, \mathrm{p}=.00\right)$ obtiene un valor de Regresión Múltiple o $\mathrm{R}^{2}$ corregida de .25 , en tanto las variables predictivas que entran a formar parte de la ecuación son: Bienestar emocional y físico subjetivo (BEFSub) $(\mathrm{t}=5.57 ; \mathrm{p}=.00)$ y Bienestar Laboral Subjetivo $($ BLSub) $(\mathrm{t}=2.99 ; \mathrm{p}=.00)$ :

$$
\mathrm{BEFObj}=1.31+.20 \mathrm{BEFSub}+.10 \mathrm{BLSub}
$$

\section{CONCLUSIONES}

La importancia de la mejora de la calidad de vida es un aspecto destacable en la sociedad. Especialmente importante es esa búsqueda en la situación que se encuentran las personas con discapacidad cuando son adultas. Afortunadamente, cada vez y con mayor frecuencia se dispone de estudios que tiene como objetivo principal tal fin.

Más concretamente, si la educación es concebida como uno de los pilares en la búsqueda de dicha calidad, se deben tener en cuenta las percepciones de los propios implicados e, incluso, de los profesionales que trabajan en la formación que reciben las personas con discapacidad.

Atendiendo a los resultados obtenidos, es importante destacar lo hallado para la dimensión de Autodeterminación. Parece que las percepciones de los profesionales sobre si una persona con discapacidad es autodeterminada se refieren a que la propia persona con discapacidad sea consciente de su propia autodeterminación. Además, se destaca igualmente la necesidad de que la persona se encuentre incluida en la sociedad y que tenga un clima familiar y social e, incluso, personal, adecuado. Pero, como afirma Peralta (2008), para lograr una mejora en estas dimensiones es necesario modificar los modelos de enseñanza 
y los estilos educativos. Los modelos educativos deben ser inclusivos y encaminados hacia un modelo de vida independiente. También es destacable que tratándose de profesionales de la educación, y concretamente de una formación profesional, la autodeterminación no se vea acompañada de la percepción de bienestar laboral.

En cuanto a la inclusión social, los resultados han mostrado que las percepciones de los profesionales sobre esta dimensión tienen como único predictor la propia percepción de la persona con discapacidad. Esto concuerda con lo descrito por Díaz (2010) sobre la necesidad de construir una sociedad inclusiva que vea a la discapacidad como una característica más de la persona y no una circunstancia excluyente para la participación en todos los procesos; incluyendo la educación como uno de los más importantes. Incluso, en esta afirmación se podría englobar el resultado que hace referencia a la percepción por parte de los profesionales del bienestar laboral, debido a que este está asociado a su homólogo en la percepción personal de las personas con discapacidad. De este modo, se repite la circunstancia que una única dimensión objetiva se describe por su misma dimensión subjetiva. Este resultado señala que la percepción de los profesionales no contempla otras dimensiones del alumnado que no se sean su propia autopercepción de inclusión social.

Sin embargo, si se habla de la dimensión de bienestar material objetivo, los profesionales perciben que para que una persona con discapacidad tenga una adecuada situación en este aspecto, no solo debe ser consciente la propia persona de esta circunstancia, sino que debe sentirse integrado en su familia y que debe tener una alta concepción de su autodeterminación.

Por otro lado, el bienestar emocional y físico percibido por los profesionales sí tiene una relación directa con el propio bienestar expresado por los estudiantes con discapacidad, y este, a su vez, se relaciona con el bienestar laboral que perciben ellos mismos. De este modo, se describe como esencial que las personas se sientan integradas en los trabajos que desarrollan; un empleo adecuado para las personas con discapacidad es fundamental para asegurar sus derechos en la sociedad (Castro y Vilá, 2014).

Finalmente, es importante destacar, teniendo en cuenta lo expuesto en párrafos anteriores, algunas cuestiones significativas. En primer lugar, hay que resaltar que todas las variables analizadas desde la perspectiva objetiva-visión de los profesionales-, tienen como variables predictivas sus homólogas en la variable subjetiva. Es decir, los profesionales encuentran que lo que opina la persona con discapacidad sobre sus propias condiciones de vida es determinante para evaluar la calidad de vida desde sus percepciones externas. Esto es significativo ya que los actuales modelos de sociedad promueven una participación de las personas con discapacidad desde un modelo de vida independiente (Díaz, 2010).

En segundo lugar, como se puede desprender de algunas afirmaciones ya realizadas, es importante destacar la necesidad de que los profesionales que forman a personas con discapacidad tengan en cuenta una mayor consideración por una educación global en la que se contemplen todas las dimensiones de las personas, para así mejorar su calidad de vida (Schalock, 2009). Además, este es uno de los objetivos fundamentales de la educación inclusiva, que tiene entre sus premisas una educación de calidad para mejorar todos los aspectos de la persona. Por lo tanto, es necesario destacar que los educadores deberían recibir formación sobre cómo atender a la globalidad de las personas, ya que como se demuestra en este trabajo, la visión de las propias personas con discapacidad no se centra única y exclusivamente en un aspecto concreto, sino que se enmarca en la confluencia de diversas dimensiones relacionadas con la calidad de vida. 
En tercer lugar, como reflexión global se sostiene que es necesario describir que los profesionales educativos que trabajan en la formación laboral de las personas con discapacidad deben conocer aquellas metodologías que permiten la flexibilidad y la inclusión de este colectivo, ya que como se ha demostrado, las percepciones subjetivas de los estudiantes con discapacidad están presentes en los indicadores de calidad de vida externos de este colectivo. Por lo tanto, metodologías como el denominado diseño universal para el aprendizaje (Sala et al., 2014), que favorecen la inclusión de todo el alumnado, y en especial de las personas con discapacidad, deberían estar presentes en los planes de formación del profesorado en Chile.

\section{REFERENCIAS BIBLIOGRÁFICAS}

Alomar, E., \& Cabré, M. (2005). El trabajo de jóvenes con Discapacidad Intelectual en entorno normalizado. Síndrome de Down, 22, 118-124.

Belmonte, M. C., \& Suñé, M. V. (2014). The transition to working life plans. An inclusive training way for people with disabilities. REOP-Revista Española de Orientación y Psicopedagogía, 25(2), 24-39.

Booth, T., \& Ainscow, M. (2002). Index for Inclusion. Developing learning and participation in schools. CSIE Mark Vaughan. Recuperado desde: http://www.eenet.org.uk/resources/docs/ Index\%20English.pdf

Burgstahler, S. (2006). Equal Access: Universal Design of Instruction. Recuperado desde http:// www.washington.edu/doit/Brochures/Academics/equal_access_udi.html

Burgstahler, S., \& Cory, R.C. (Eds.). (2008). Universal Design in Higher Education: From Principles to Practice. Cambirdge, MA.: Harvard Education Press.

Castellana, M., \& Sala, I. (2005). La universidad ante la diversidad en el aula. Aula Abierta, 85, 57-84.

Castro, L., Cerda, G., Vallejos, V., Zúñiga, D., \& Cano, R. (2016). Calidad de Vida de personas con discapacidad intelectual en centros de formación laboral. Avances en Psicología latinoamericana, 34(1), 175-186.

Castro, M., \& Vilá, M. (2014). Los planes de transición al trabajo: una opción formativa inclusiva para personas con discapacidad. REOP-Revista Española de Orientación y Psicopedagogía, 25(2), 24-39.

Coll, C., \& Miras, M. (2001). Diferencias individuales y atención a la diversidad. En A. Marchesi, C. Coll y J. Palacios (Comps.), Desarrollo psicológico y Educación (Vol. 2, pp. 331-356). Madrid: Alianza Editorial.

Díaz, E. (2010). Ciudadanía, identidad y exclusión social de las personas con discapacidad. Política $y$ sociedad, 47(1), 115-135.

Echeita, E. (2008). Inclusión y exclusión educativa. "Voz y quebranto". REICE-Revista Electrónica Iberoamericana sobre Calidad, Eficacia y Cambio en Educación, 6(2), 9-18

Echeita, G., \& Simón, C. (2007). La contribución de la educación escolar a la calidad de vida de las personas con discapacidad. Ante el desafío de su inclusión social. En R. de Lorenzo y L. C. Pérez Bueno (Dirs.), Tratado sobre discapacidad (pp. 1103-1133). Navarra: Aranzadi.

European Association for Quality Assurance in Higher Education. (2005). Criterios y Directrices para la Garantía de Calidad en el Espacio Europeo de Educación Superior. Helsinki: ENQA.

Díez, E., Alonso, A., Verdugo, M.A., Campo, I., Sancho, I., Sánchez, S., ... Moral, E. (2011). Espacio Europeo de Educación Superior: estándares e indicadores de buenas prácticas para la atención a estudiantes universitarios con discapacidad. Salamanca: Universidad de Salamanca.

Dukes, L. L. (2001). The process: Development of AHEAD Program Standards. Journal of Postsecondary Education and Disability, 14, 62-80. 
Estudios Pedagógicos XLII, $\mathrm{N}^{\circ}$ 2: 39-49, 2016

PERCEPCIÓN DE LA CALIDAD DE VIDA EN PERSONAS CON DISCAPACIDADY SU RELACIÓN CON LA EDUCACIÓN

Dukes, L. L. (2006). The Process: Development of the revised AHEAD Program Standards and Performance Indicators. Journal of Postsecondary Education and Disability, 19, 5-15.

Echeita, G. (2001). Claves e indicios para la valoración de Integración / Inclusión en España. En M. Verdugo y F. Jordán de Urríes Vega (Coords.), Apoyos, autodeterminación y calidad de vida (pp. 161-176). Salamanca: Amarú.

Gandasegui, V. D., \& Méndez, F. C. (2014). Necesidades formativas del profesorado de secundaria en la docencia a alumnos con discapacidad. RISE-International Journal of Sociology of Education, 3(3), 192-217.

Gobierno de Chile. Ley $N^{\circ} 19.284$ que establece las normas para la plena integración social de personas con discapacidad. Recuperado desde: http://www.crececontigo.gob.cl/wp-content/ uploads/2010/01/Ley_de_Integracion_Social_de_las_Personas_con_Discapacidad_-_19.284.pdf

Gómez, L.E., Verdugo, M.A., \& Arias, B. (2015). Validity and reliability of the INICO-FEAPS Scale: An Assessment of Quality of Life for People with Intellectual and Developmental Disabilities. Research in Intellectual and Developmental Disabilities, 36, 600-610. doi:10.1016/j. ridd.2014.10.049

Gómez, L. E., Verdugo, M. A., Arias, B., \& Arias, V. (2011). A comparison of alternative models of individual quality of life for social service recipients. Social Indicators Research, 101(1), 109125.

Gómez-Vela, M., \& Verdugo, M.A. (2004). El cuestionario de evaluación de la calidad de vida de alumnos de educación secundaria obligatoria: descripción, validación inicial y resultados obtenidos tras su aplicación en una muestra de adolescentes con discapacidad y sin ella. Siglo Cero, 35(4), 5-17.

González, F., \& Araneda, P. (2005). Integración de las personas con discapacidad en la educación superior en Chile. Santiago: IESALC /UNESCO.

Jordán de Urríes, F.B., \& Verdugo, M.A. (2003). Variables del apoyo, la persona y el empleo. Breve explicación de la investigación desarrollada. En M.A. Verdugo y F.B. Jordán de Urríes (Eds.), Investigación, innovación y cambio (pp. 121-145). Salamanca: Amarú.

Luckasson, R., Coulter, D., Polloway, E., Reiss, S., Schalock, R., Snell M. E., ... Stark, J. (1992). Mental retardation: Classification and systems of supports (9th ed.). Washington, DC: American Association.

Martín, E., y Mauri, T. (Coords.). (1997). La atención a la diversidad en la Educación Secundaria. Barcelona: ICCE/Horsori.

Martínez, R., De Haro, R., \& Escarbajal, A. (2010). Una aproximación a la educación inclusiva en España. Revista Educación Inclusiva, 3(1), 149-164.

Montero, I., \& León, O. G. (2007). A guide for naming research studies in Psychology. International Journal of Clinical and Health Psychology, 7(3), 847-862.

Pallisera, M., (2011). La inclusión laboral y social de los jóvenes con discapacidad intelectual. El papel de la escuela. Revista Interuniversitaria de Formación de Profesorado, 25(1), 185-200.

Pallisera, M., Fullana, J., \& Vila, M. (2010). La inserción laboral de personas con discapacidad. Desarrollo de tres investigaciones acerca de los factores favorecedores de los procesos de inserción. Revista de Investigación Educativa, 23(2), 295-313.

Peralta, F. (2008). Educar en Autodeterminación: profesores y padres como principales agentes educativos. Educación y diversidad. Anuario internacional de investigación sobre Discapacidad e Interculturalidad, 2, 151-156.

Sala, I., Sánchez, S., Giné, C., \& Díez, E. (2014). Análisis de los distintos enfoques del paradigma del diseño universal aplicado a la educación. Revista Latinoamericana de Educación Inclusiva, $8(1), 143-152$.

Samaniego, P. (2009). Discapacidad y educación en Latinoamérica. En P. Samaniego (Dir.), Personas con discapacidad y acceso a servicios educativos en Latinoamérica (pp. 171-322). Madrid: CERMI. 
Sánchez, S. (2013). Aplicación del paradigma del diseño universal en la educación universitaria: implantación de estudios y percepción sobre inclusión de medidas curriculares (Tesis doctoral inédita). Universidad de Salamanca. Salamanca.

Seda, J. A. (2008). Discapacidad y educación universitaria. En C. Eroles y H. Fiamberti (Eds.), Los derechos de las personas con discapacidad (pp. 191-199). Buenos Aires: Eudeba.

Schalock, R. L. (2009). La nueva definición de discapacidad intelectual, apoyos individuales y resultados personales. Siglo Cero, 40(1), 22-39.

Schalock, R. L., \& Verdugo, M. A. (2002/2003). Quality of life for human service practitioners. Washington, DC: American Association on Mental Retardation.

Schalock, R. L., \& Verdugo, M. A. (2007). El concepto de calidad de vida en los servicios y apoyos para personas con discapacidad intelectual. Siglo Cero, 38, 21-36.

Schalock, R. L. \& Verdugo, M. A. (2008). Quality of life: from concept to application in the field of intellectual disabilities. Evaluation \& Program Planning, 31, 181-190.

Schelly, C., Davies, P., \& Spooner, C. (2011). Student perceptions of faculty implementation of universal design for learning. Journal of Postsecondary Education and Disability, 24(1), 17-30.

Thompson, J., Hughes, C., Schalock, R., Silverman, W., Tasse, M., Bryant, B., ... Campbell, E. (2003). Integrando los apoyos en la evaluación y planificación. Siglo Cero, 34(2), 36-55.

Verdugo, M. A. (2006). Cómo mejorar la calidad de vida de las personas con discapacidad. Salamanca: Amarú.

Verdugo, M.A., Gómez, L., \& Arias, B. (2007). La Escala Integral de Calidad de Vida. Desarrollo y Estudio Preliminar de sus Propiedades Psicométricas. Siglo Cero, 38(4), 37-56.

Verdugo, M.A., Gómez, L.E., \& Arias, B. (2009). La Escala FUMAT de evaluación de la calidad de vida de personas mayores y con discapacidad. Salamanca: INICO.

Verdugo, M. A., Gómez, L. E., Arias, B., \& Navas, P. (2010). Aplicación del paradigma de calidad de vida: construcción de escalas de evaluación e investigación. En M. A. Verdugo, M. Crespo y T. Nieto (Coords.), Aplicación del paradigma de calidad de vida (pp. 19-38). Salamanca: INICO.

Verdugo, M. A., Gómez, L. E., Schalock, R. L., \& Arias, B. (2011). The Integral Quality of Life Scale: Development, Validation, and Use. En R. Kober (Ed.), Quality of life for people with intellectual disability (pp. 47-60). Nueva York: Springer.

Wang, M., Schalock, R. L., Verdugo, M.A., \& Jenaro, C. (2010). Analyzing the factor structure and hierarchical nature of the quality of life construct. American Journal on Intellectual and Developmental Disabilities, 115, 218-233. 
